Supporting Information

\title{
Synthesis and Metal Complex Formation of Bis(terpyridine) Derivative Having Phenylacetylene Framework
}

\author{
Tomoyuki Tokizaki,a Takuya Toyoshima, ${ }^{a, b}$ Soichiro Watanabe ${ }^{a, b}$ \\ a Department of Biomolecular Science, Faculty of Science, Toho University, Miyama 2-2-1, Funabashi, \\ Chiba, 274-8510 Japan \\ Fax +81(47)4721298; E-mail: soichiro@biomol.sci.toho-u.ac.jp \\ b Research Center for Materials with Integrated Properties, Toho University, Miyama 2-2-1, \\ Funabashi, Chiba, 274-8510 Japan
}

Table of Contents

Full Experimental Detail for Compounds 3, 4, and 6-8 S2-S5

UV/Vis and NMR Titration Experiment S5-S6

NMR Spectra of Compound $3 \quad$ S7-S8

NMR Spectra of Compound $4 \quad$ S9-S10

NMR Spectra of Compound $6 \quad$ S11-S12

NMR Spectra of Compound $7 \quad$ S13-S14

NMR Spectra of Compound $8 \quad$ S15-S16

NMR Spectra of Compound $1 \quad$ S17-S21

UV/Vis Titration of Compound 1 with $\mathrm{CoCl}_{2} \quad$ S22

UV/Vis Titration of Compound 1 with $\mathrm{FeCl}_{2} \quad$ S23

NMR Titration of Compound 1 with $\mathrm{ZnCl}_{2} \quad \mathrm{~S} 24$

ESI-MS Spectra of Compound 1 with $\mathrm{ZnCl}_{2} \quad$ S25 
Experimental

Melting points were determined on a Yanaco micro melting point apparatus. All melting points were uncorrected. Preparative gel permeation chromatography was performed by LC-9201 (Japan Analytical Industry) with JAIGEL $1 \mathrm{H}+2 \mathrm{H}$ columns with $\mathrm{CHCl}_{3}$ as solvent. ${ }^{1} \mathrm{H}$ and ${ }^{13} \mathrm{C} \mathrm{NMR}$ as well as $\mathrm{HH}$ COSY and HSQC spectra were measured in $\mathrm{CDCl}_{3}$ with a Bruker Avance 300 and 400 spectrometers using tetramethylsilane as an internal standard. UV/Vis spectra were recorded on a HITACHI U-3010 spectrophotometer. High resolution mass spectra were recorded on a Thermo Fisher Exactive at Center for Analytical Instrumentation, Chiba University. Elemental analyses were performed by the Instrument Analysis Center in Faculty of Sciences, Toho University.

2-Bromo-5-[(3-iodophenyl)ethynyl]pyridine (3)

To a solution of 1, 3-diiodobenzene (907 mg, $2.75 \mathrm{mmol}$ ), CuI (15 mg, 0.079 $\mathrm{mmol})$, and $\mathrm{Pd}\left(\mathrm{PPh}_{3}\right)_{4}(35 \mathrm{mg}, 0.030 \mathrm{mmol})$ in degassed triethylamine $(15 \mathrm{ml})$ was added a solution of 2-bromo-5-ethynylpyridine $2^{\text {[S1] }}$ (200 mg, $\left.1.10 \mathrm{mmol}\right)$ in degassed triethylamine $(2.5 \mathrm{ml})$ and the reaction mixture was stirred overnight under reflux. After cooled to room temperature, $\mathrm{Et}_{2} \mathrm{O}$ was added to the reaction mixture, then the precipitate was filtered off. The solvent was evaporated under reduced pressure and the residue was separated by column chromatography $\left(\mathrm{SiO}_{2} /\right.$ hexane:EtOAc $\left.=9: 1\right)$ to afford compound 3 (310 mg, $0.81 \mathrm{mmol}, 74 \%$ ) as white solid.

$\mathrm{Mp} 120-122^{\circ} \mathrm{C}$.

${ }^{1} \mathrm{H} \mathrm{NMR}\left(300 \mathrm{MHz} \mathrm{CDCl}_{3}\right): \delta 8.50(\mathrm{~d}, J=2.0 \mathrm{~Hz}, 1 \mathrm{H}), 7.90(\mathrm{t}, J=2.0 \mathrm{~Hz}, 1 \mathrm{H})$, 7.71(d, $J=8.1 \mathrm{~Hz}, 1 \mathrm{H}), 7.64(\mathrm{dd}, J=8.1,2.0 \mathrm{~Hz}, 1 \mathrm{H}), 7.50(\mathrm{~d}, J=8.1 \mathrm{~Hz}, 2 \mathrm{H})$, 7.11(t, $J=8.1 \mathrm{~Hz}, 1 \mathrm{H})$.

${ }^{13} \mathrm{C} \mathrm{NMR}\left(75 \mathrm{MHz}, \mathrm{CDCl}_{3}\right): \delta 152.4,141.5,140.5,140.2,138.1$ 130.8, 130.0, $127.8,124.2,119.3,93.8,92.1,86.0$.

HRMS-ESI $m / z[\mathrm{M}+\mathrm{H}]^{+}$calcd for $\mathrm{C}_{13} \mathrm{H}_{8} \mathrm{BrIN}$ : 383.8873; found: 383.8879 . 
5,5'-(\{[(2,5-dibutoxy-1,4-phenylene) bis(ethyne-2,1-diyl) $]$ bis(3,1-phenylene) $\}$ bi s(ethyne-2,1-diyl))bis(2-bromopyridine) (4)

To a solution of 1,4-dibutoxy-2,5-diethynylbenzene ${ }^{[\mathrm{S} 2]}(440 \mathrm{mg}, 1.63 \mathrm{mmol}$ ), $\mathrm{CuI}(16 \mathrm{mg}, 0.084 \mathrm{mmol})$, and $\mathrm{Pd}\left(\mathrm{PPh}_{3}\right)_{4}(85 \mathrm{mg}, 0.16 \mathrm{mmol})$ in degassed triethylamine $(10 \mathrm{ml})$ was added a solution of compound $3(1.25 \mathrm{~g}, 3.26$ mmol) in dehydrated THF (5 ml) under argon atmosphere and the reaction mixture was stirred overnight under reflux. After cooled to room temperature, the solvent was evaporated under reduced pressure. The residue was dissolved in $\mathrm{CH}_{2} \mathrm{Cl}_{2}$ and $\mathrm{CH}_{3} \mathrm{Cl}$ and the solution was washed with saturated aqueous ammonium chloride solution, water, and brine. The organic layer was dried over anhydrous $\mathrm{MgSO}_{4}$, filtered, and the solvent was evaporated under reduced pressure. The residue was separated by column chromatography $\left(\mathrm{SiO}_{2} / \mathrm{CH}_{2} \mathrm{Cl}_{2}\right)$ to afford compound $4(1.04 \mathrm{~g}, 1.34 \mathrm{mmol}$, $82 \%)$ as yellow solid.

Mp $174-177^{\circ} \mathrm{C}$.

${ }^{1} \mathrm{H} \mathrm{NMR}\left(300 \mathrm{MHz}, \mathrm{CDCl}_{3}\right): \delta 8.52(\mathrm{~d}, J=2.0 \mathrm{~Hz}, 2 \mathrm{H}), 7.71(\mathrm{~s}, 2 \mathrm{H}), 7.65(\mathrm{dd}$, $J=8.2,2.0 \mathrm{~Hz}, 2 \mathrm{H}), 7.55-7.48(\mathrm{~m}, 6 \mathrm{H}), 7.37(\mathrm{t}, J=7.7 \mathrm{~Hz}, 2 \mathrm{H}), 7.02(\mathrm{~s}, 2 \mathrm{H})$, $4.05(\mathrm{t}, J=6.4 \mathrm{~Hz}, 4 \mathrm{H}), 1.90-1.81(\mathrm{~m}, 4 \mathrm{H}), 1.66-1.53(\mathrm{~m}, 4 \mathrm{H}), 1.02(\mathrm{t}, J=7.4 \mathrm{~Hz}$, $6 \mathrm{H})$.

${ }^{13} \mathrm{C} \mathrm{NMR}\left(75 \mathrm{MHz}, \mathrm{CDCl}_{3}\right): \delta 153.7,152.4,140.5,134.6,132.0,131.3,128.7$, $127.7,125.5,124.1,122.5,119,5,116.9,113.9,93.8,93.1,86.9,85.3,69.4$, $31.4,19.3,13.9$.

HRMS-ESI $m / z[\mathrm{M}+\mathrm{H}]^{+}$calcd for $\mathrm{C}_{44} \mathrm{H}_{35} \mathrm{Br}_{2} \mathrm{~N}_{2} \mathrm{O}_{2}: 781.1071$; found 781.1060 .

2-bromo-5-(\{4-[(tert-butyldimethylsilyl)oxy]phenyl\}ethynyl)pyridine(6)

A solution of 2-bromo-5-ethynylpyridine $2^{[\mathrm{S} 1]}(1.82 \mathrm{~g}, 10.0 \mathrm{mmol})$, compound $5^{\text {[S3] }}(3.34 \mathrm{~g}, 10.0 \mathrm{mmol}), \mathrm{CuI}(57 \mathrm{mg}, 0.30 \mathrm{mmol})$, and $\mathrm{Pd}\left(\mathrm{PPh}_{3}\right)_{4}(346 \mathrm{mg}$, $0.30 \mathrm{mmol})$ in degassed triethylamine $(20 \mathrm{ml})$ was stirred at room temperature for $80 \mathrm{~min}$ under argon atmosphere. The reaction mixture was passed through a small amount of $\mathrm{SiO}_{2}$ to remove precipitate and the solvent was evaporated under reduced pressure. The residue was separated by 
column chromatography $\left(\mathrm{SiO}_{2} /\right.$ Hexane: $\left.\mathrm{Et}_{2} \mathrm{O}=11: 1\right)$ to afford compound 6 (2.10 g, $5.41 \mathrm{mmol}, 54 \%)$ as white solid.

$\operatorname{Mp} 53-55^{\circ} \mathrm{C}$.

${ }^{1} \mathrm{H}$ NMR (300 $\left.\mathrm{MHz} \mathrm{CDCl}_{3}\right): \delta 8.48(\mathrm{~d}, J=2.3 \mathrm{~Hz}, 1 \mathrm{H}), 7.62(\mathrm{dd}, J=8.2,2.3 \mathrm{~Hz}$, $1 \mathrm{H}), 7.46(\mathrm{~d}, J=8.2 \mathrm{~Hz}, 1 \mathrm{H}), 7.41(\mathrm{~d}, J=8.6 \mathrm{~Hz}, 2 \mathrm{H}), 6.83(\mathrm{~d}, J=8.6 \mathrm{~Hz}, 2 \mathrm{H})$, $0.97(\mathrm{~s}, 9 \mathrm{H}), 0.20(\mathrm{~s}, 6 \mathrm{H})$.

${ }^{13} \mathrm{C} \mathrm{NMR}\left(75 \mathrm{MHz} \mathrm{CDCl}_{3}\right): \delta 156.7,152.2,140.6,140.4,133.2,127.6,120.4$, $120.1,114.9,94.2,83.7,25.6,18.2,-4.4$.

Anal calcd for $\mathrm{C}_{19} \mathrm{H}_{22}$ BrNOSi: C, 58.76; H, 5.71; N, 3.61; found C, 58.51; H, $5.66 ; \mathrm{N}, 3.56$.

5-(\{4-[(tert-butyldimethylsilyl)oxy]phenyl\}ethynyl)-2-(tributylstannyl)pyridi ne (7)

To a solution of compound $6(1.55 \mathrm{~g}, 4.00 \mathrm{mmol})$ in dehydrated THF (15 ml) was added $n \mathrm{BuLi}(2.5 \mathrm{M}$ in THF, $1.76 \mathrm{ml}, 4.40 \mathrm{mmol})$ at $-78{ }^{\circ} \mathrm{C}$ under argon atmosphere and the reaction mixture was stirred for $20 \mathrm{~min}$. To the reaction mixture was added $\mathrm{Bu}_{3} \mathrm{SnCl}(1.20 \mathrm{ml}, 4.40 \mathrm{mmol})$ and stirred at room temperature for $30 \mathrm{~min}$. Saturated aqueous ammonium chloride solution was added to the reaction mixture, then the solution was extracted with $\mathrm{Et}_{2} \mathrm{O}$. The organic layer was washed with water and brine, and dried over anhydrous $\mathrm{MgSO}_{4}$, filtered, and the solvent was evaporated under reduced pressure. The residue was separated by column chromatography $\left(\mathrm{SiO}_{2}\right.$ with $10 \mathrm{w} / \mathrm{w} \%$ of $\mathrm{K}_{2} \mathrm{CO}_{3} /$ Hexane:Et $\left.{ }_{2} \mathrm{O}=11: 1\right)$ to afford compound 7 (1.71 g, 2.86 mmol, $72 \%$ ) as orange oil.

${ }^{1} \mathrm{H} \mathrm{NMR}\left(300 \mathrm{MHz}, \mathrm{CDCl}_{3}\right): \delta 8.84(\mathrm{~d}, J=2.1 \mathrm{~Hz}, 1 \mathrm{H}), 7.58(\mathrm{dd}, J=7.7,2.1 \mathrm{~Hz}$, $1 \mathrm{H}), 7.42(\mathrm{~d}, J=8.6 \mathrm{~Hz}, 2 \mathrm{H}), 7.39(\mathrm{~d}, J=7.7 \mathrm{~Hz}, 1 \mathrm{H}), 6.82(\mathrm{~d}, J=8.6 \mathrm{~Hz}, 2 \mathrm{H})$, $1.61-1.51(\mathrm{~m}, 6 \mathrm{H}), 1.39-1.27(\mathrm{~m}, 6 \mathrm{H}), 1.16-1.10(\mathrm{~m}, 6 \mathrm{H}), 0.99(\mathrm{~s}, 9 \mathrm{H}), 0.88(\mathrm{t}$, $J=7.2 \mathrm{~Hz}, 9 \mathrm{H}), 0.20(\mathrm{~s}, 6 \mathrm{H})$.

${ }^{13} \mathrm{C}$ NMR (75 MHz, $\left.\mathrm{CDCl}_{3}\right): \delta 173.6,156.3,152.2,135.1,133.1,131.4,120.3$, $118.8,115.5,92.4,85.5,29.1,27.3,25.6,18.2,13.7,9.9,-4.4$.

HRMS-ESI $m / z[\mathrm{M}+\mathrm{H}]+{ }^{+}$calcd for $\mathrm{C}_{31} \mathrm{H}_{50} \mathrm{NOSiSn}: 600.2670$; found 600.2678. 
6'-bromo-5-((4-((tert-butyldimethylsilyl)oxy)phenyl)ethynyl)-2,2'-bipyridine (8)

To a solution of compound 7 (1.71 g, $2.86 \mathrm{mmol})$, CuI (70 mg, $0.37 \mathrm{mmol})$, and $\mathrm{Pd}\left(\mathrm{PPh}_{3}\right)_{4}(345 \mathrm{mg}, 0.29 \mathrm{mmol})$ in dehydrated toluene $(20 \mathrm{ml})$ was added a solution of 2-bromo-6-iodopyridine ${ }^{[\mathrm{S} 4]}(893 \mathrm{mg}, 3.15 \mathrm{mmol})$ in dehydrated THF (5 ml) under argon atmosphere and the reaction mixture was stirred overnight under reflux. After cooled to room temperature, the solvent was evaporated under reduced pressure. The residue was separated by column chromatography $\left(\mathrm{SiO}_{2}\right.$ with $10 \mathrm{w} / \mathrm{w} \%$ of $\mathrm{K}_{2} \mathrm{CO}_{3} /$ Hexane: $\left.\mathrm{Et}_{2} \mathrm{O}=9: 1\right)$ to afford compound 8 (897 mg, $1.93 \mathrm{mmol}, 68 \%$ ) as white solid.

$\operatorname{Mp} 149-151{ }^{\circ} \mathrm{C}$.

${ }^{1} \mathrm{H}$ NMR (300 $\left.\mathrm{MHz} \mathrm{CDCl}_{3}\right): \delta 8.78(\mathrm{~d}, J=2.0 \mathrm{~Hz}, 1 \mathrm{H}), 8.41(\mathrm{dd}, J=8.0,2.0 \mathrm{~Hz}$, $2 \mathrm{H}), 7.92(\mathrm{dd}, J=8.0,2.0 \mathrm{~Hz}, 1 \mathrm{H}), 7.70(\mathrm{t}, J=8.0 \mathrm{~Hz}, 1 \mathrm{H}), 7.51(\mathrm{~d}, J=8.0 \mathrm{~Hz}, 1 \mathrm{H})$, 7.48(d, $J=8.5 \mathrm{~Hz}, 2 \mathrm{H}), 6.86(\mathrm{~d}, J=8.5 \mathrm{~Hz}, 2 \mathrm{H}), 1.01(\mathrm{~s}, 9 \mathrm{H}), 0.24(\mathrm{~s}, 6 \mathrm{H})$.

${ }^{13} \mathrm{C}$ NMR (75 $\left.\mathrm{MHz} \mathrm{CDCl}_{3}\right): \delta 156.8,156.6,152.8,151.5,141.7,139.22$, 139.17, 133.3, 128.1, 121.3, 120.7, 120.4, 119.9, 115.2, 94.2, 85.2, 25.6, 18.2, -4.4 .

HRMS-ESI $m / z[\mathrm{M}+\mathrm{H}]+$ calcd for $\mathrm{C}_{24} \mathrm{H}_{26} \mathrm{BrN}{ }_{2} \mathrm{OSi}$ : 465.0988; found 465.0992.

UV/Vis titration

UV/Vis spectra were measured using a solution of compound 1 in $\mathrm{CHCl}_{3}$ $(4.50 \mu \mathrm{M}, 3 \mathrm{ml})$. Solutions of $\mathrm{ZnCl}_{2}, \mathrm{CoCl}_{2}$, and $\mathrm{FeCl}_{2}(0.14 \mathrm{mM})$ were added to the bis(terpyridine) solution. UV/Vis spectra were measured after addition of a $20 \mu \mathrm{l}$ of metal chloride solution, and repeated this procedure until 2 eq. of metal chlorides were added.

NMR titration

NMR spectra were measured using a solution of compound 1 (12.08 mg, 8.67 $\mu \mathrm{mol})$ in $\mathrm{CDCl}_{3}$. A Solution of $\mathrm{ZnCl}_{2}$ in $\mathrm{CD}_{3} \mathrm{OD}(86.7 \mathrm{mM})$ was added to the 
bis(terpyridine) solution. NMR spectra were measured after addition of a 20 $\mu \mathrm{l}$ of $\mathrm{ZnCl}_{2}$ solution, and repeated this procedure until 2 eq. of $\mathrm{ZnCl}_{2}$ was added.

\section{References}

S1. Grave, C.; Lentz, D.; Schäfer, A.; Samori, P.; Rabe, J. P.; Franke, P.;

Schlüter, A. D. Shape-Persistant Macrocycles with Terpyridine Units:

Synthesis, Characterization, and Structure in the Crystal. J. Am. Chem. Soc. 2003, 125, 6907.

S2. Harriman, A.; Mallon, L. J.; Elliot, K. J.; Haefele, A.; Ulrich, G.; Ziessel, R. Length Dependence for Intramolecular Energy Transfer in Three- and Four-Color Donor-Spacer-Acceptor Arrays. J. Am. Chem. Soc. 2009, 131, 13375.

S3. Ogawa, T.; Ohta, K.; Iijima, T.; Suzuki, T.; Ohta, S.; Endo, Y. Synthesis and biological evaluation of p-carborane bisphenols and their derivatives: Structure-activity relationship for estrogenic activity. Bioorg. Med. Chem. 2009, 17, 1109.

S4. Trécourt, F.; Breton, G.; Bonnet, V.; Mongin, F.; Marsais, F.; Quéguiner, G. New Syntheses of Substituted Pyridines via Bromine-Magnesium Exchange. Tetrahedron 2000, 56, 1349. 
Figure S1. ${ }^{1} \mathrm{H}$ NMR Spectra of Compound 3

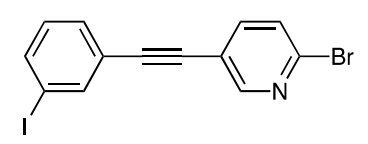

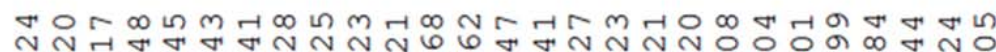

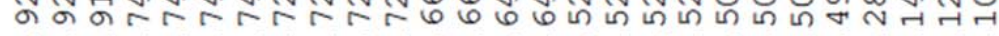
-

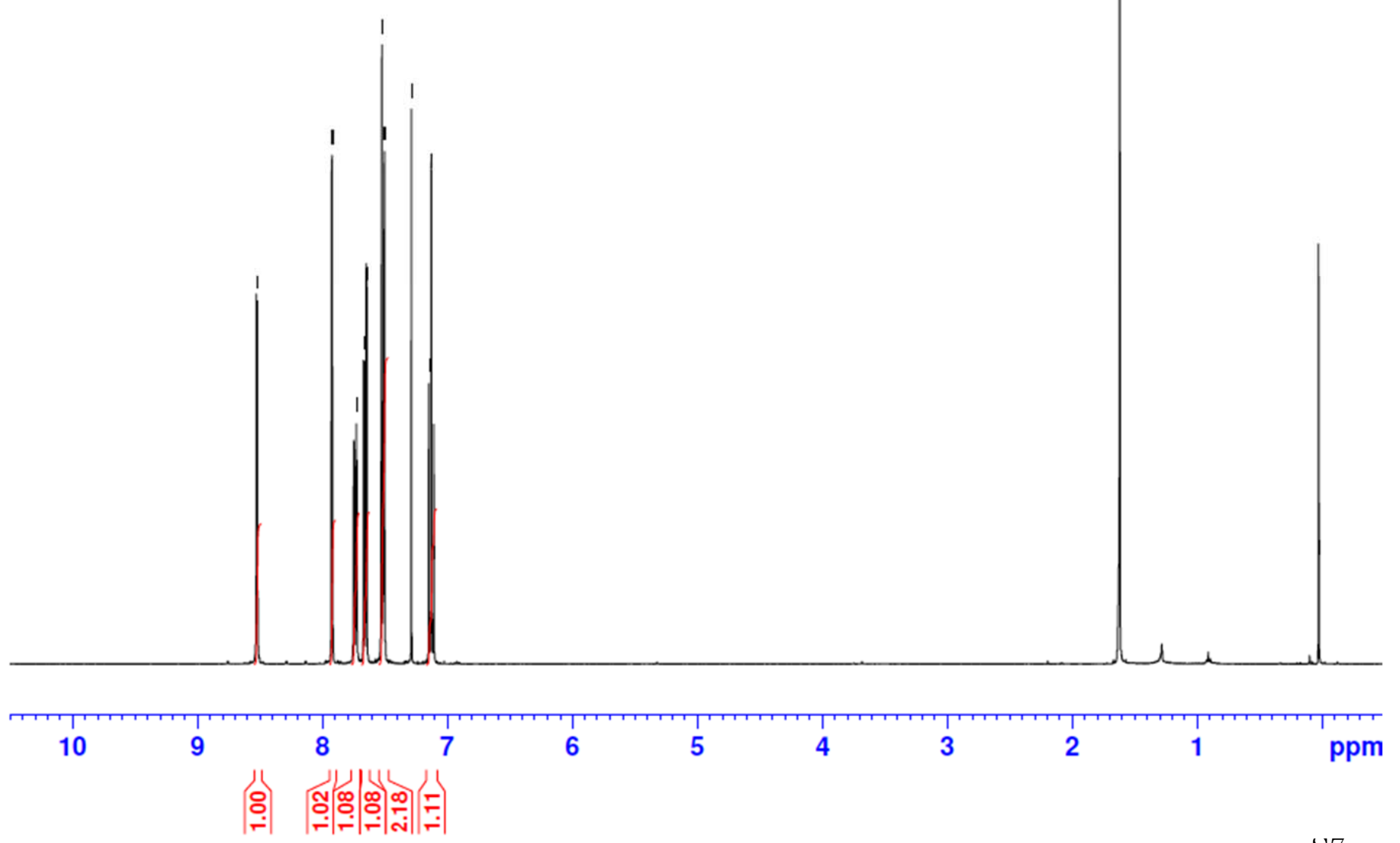


Figure S2. ${ }^{13} \mathrm{C}$ NMR Spectra of Compound 3
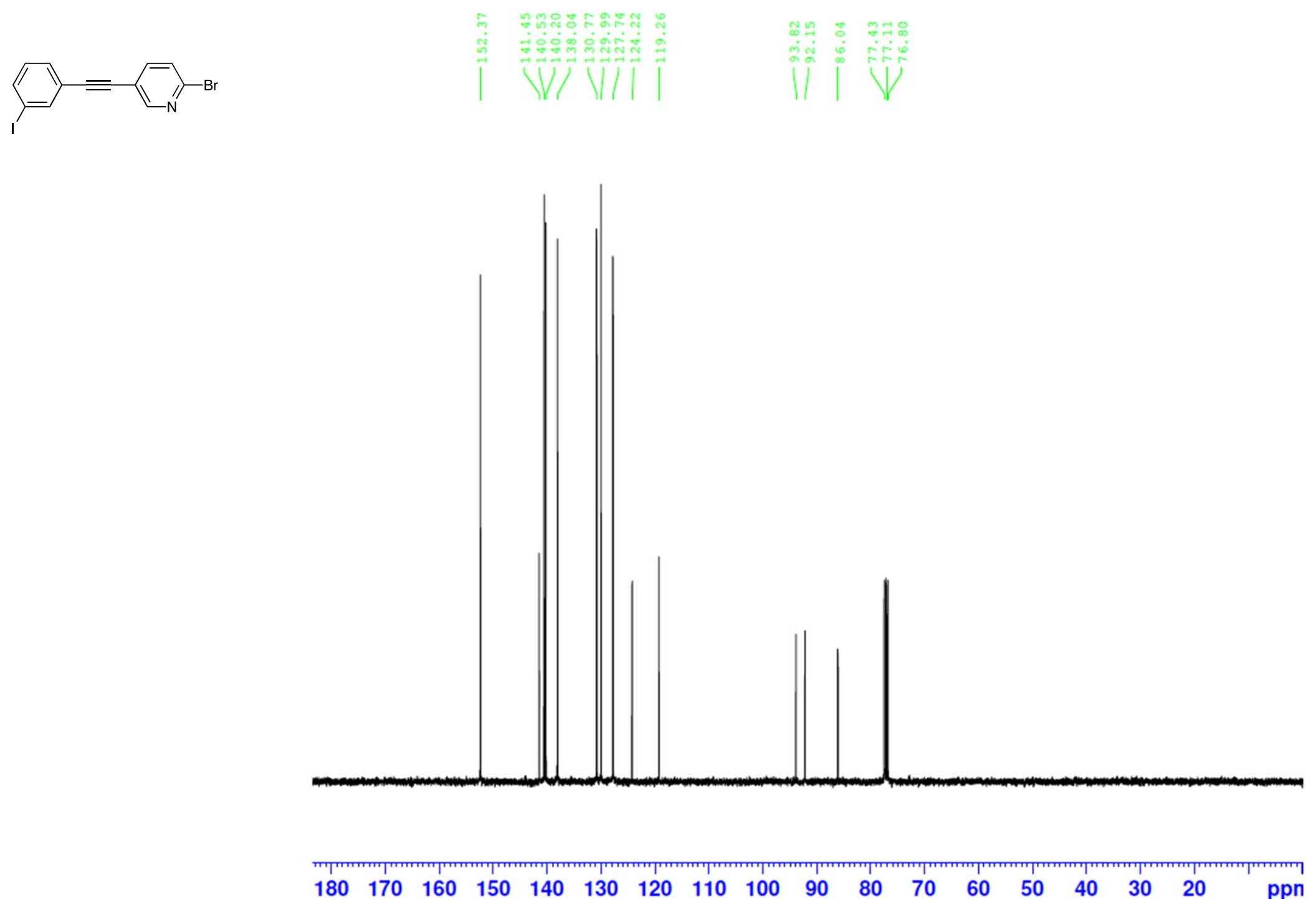
Figure S3. ${ }^{1} \mathrm{H}$ NMR Spectra of Compound 4

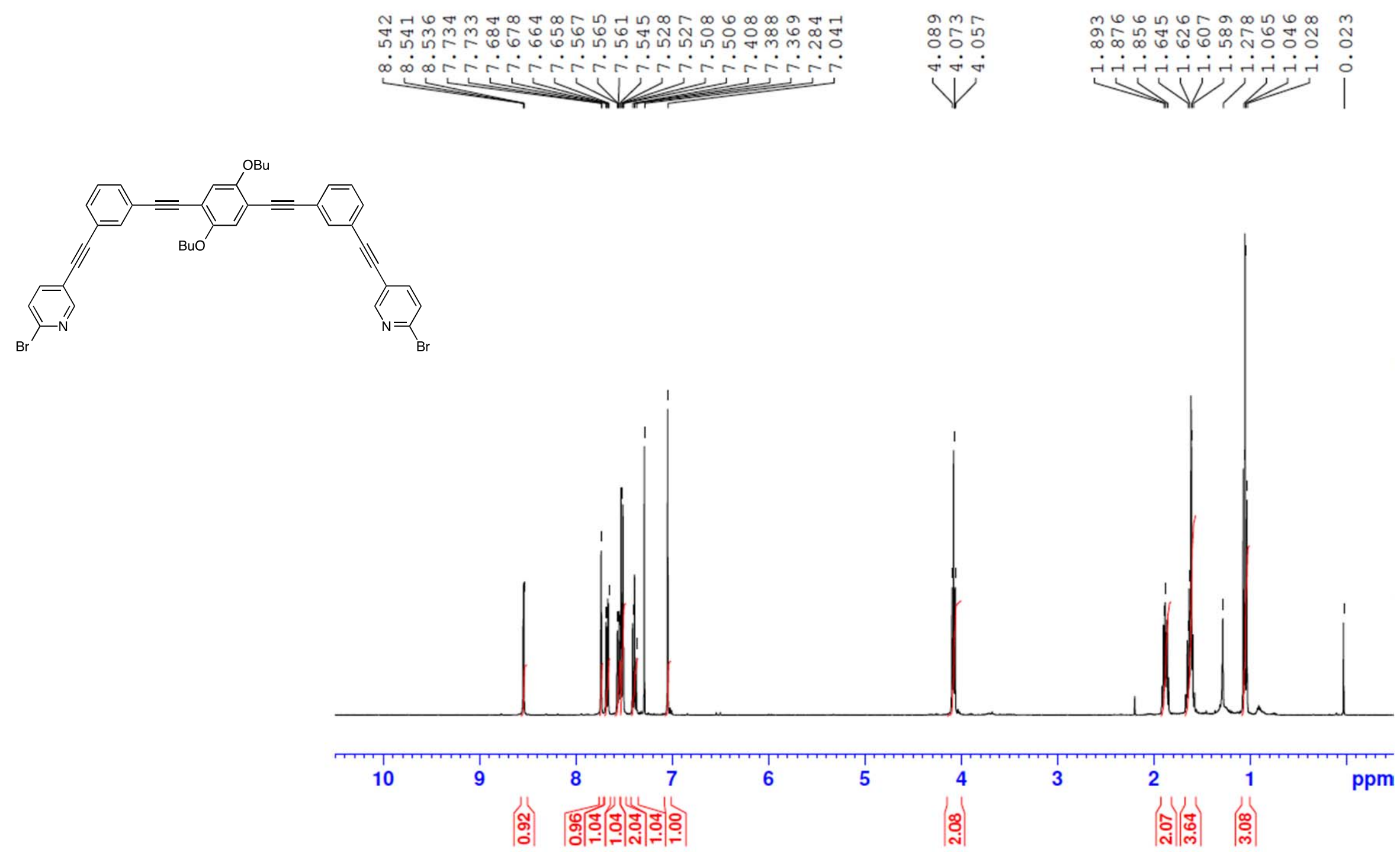


Figure S4. ${ }^{13} \mathrm{C}$ NMR Spectra of Compound 4
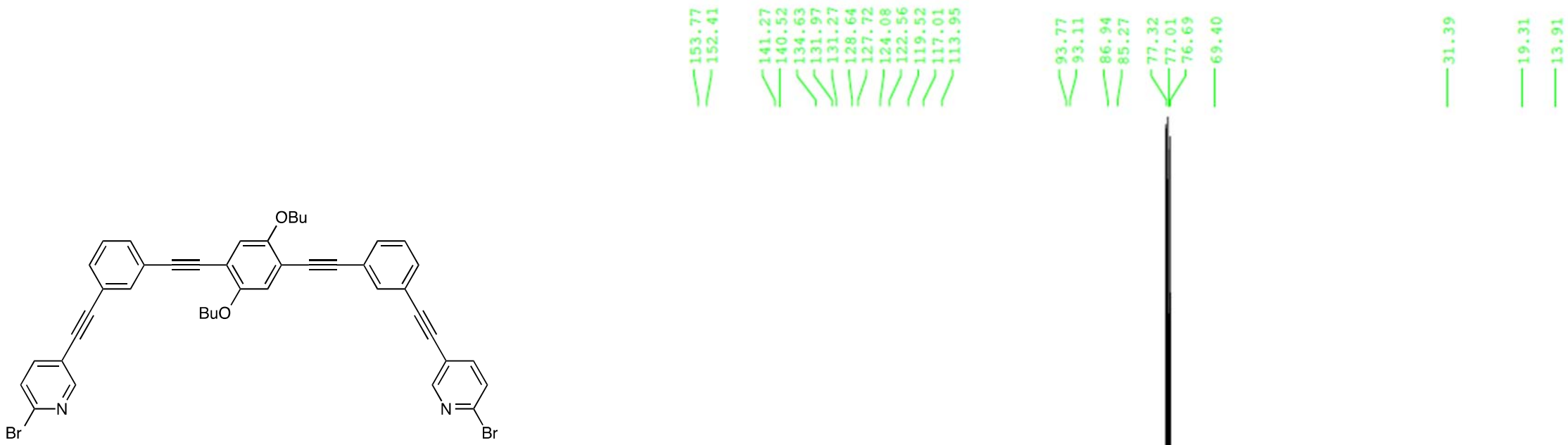

$\overbrace{\mathrm{Br}} \mathrm{N}$

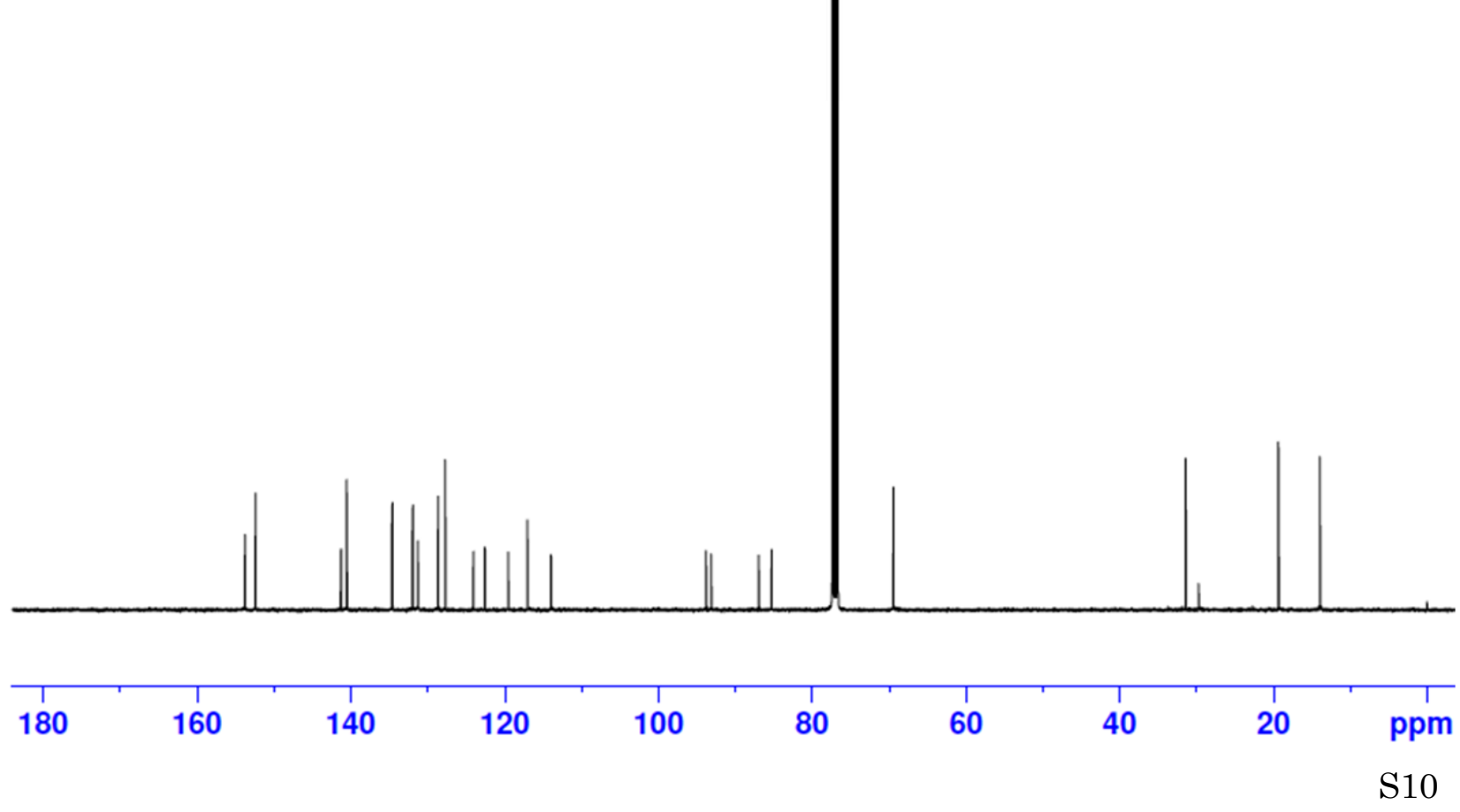


Figure S5. ${ }^{1} \mathrm{H}$ NMR Spectra of Compound $\mathbf{6}$

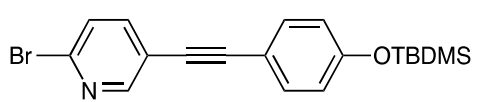

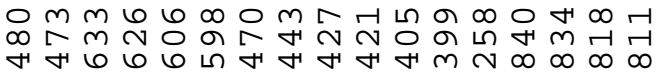

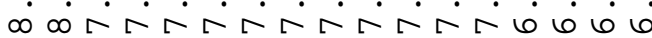
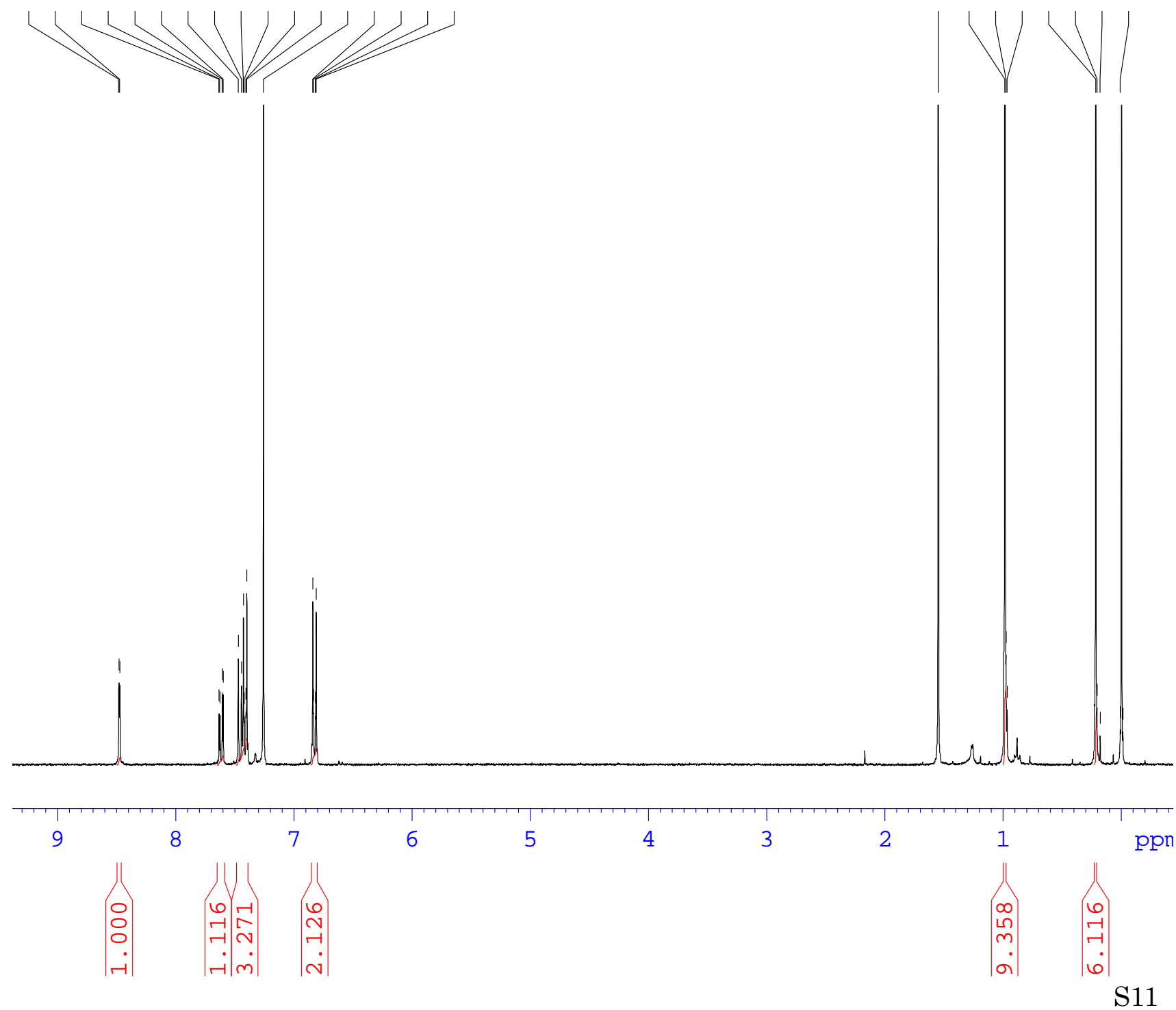

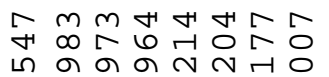

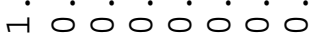


Figure S6. ${ }^{13} \mathrm{C}$ NMR Spectra of<smiles>CC(C)(O)Oc1ccc(C#Cc2ccc(Br)nc2)cc1</smiles>

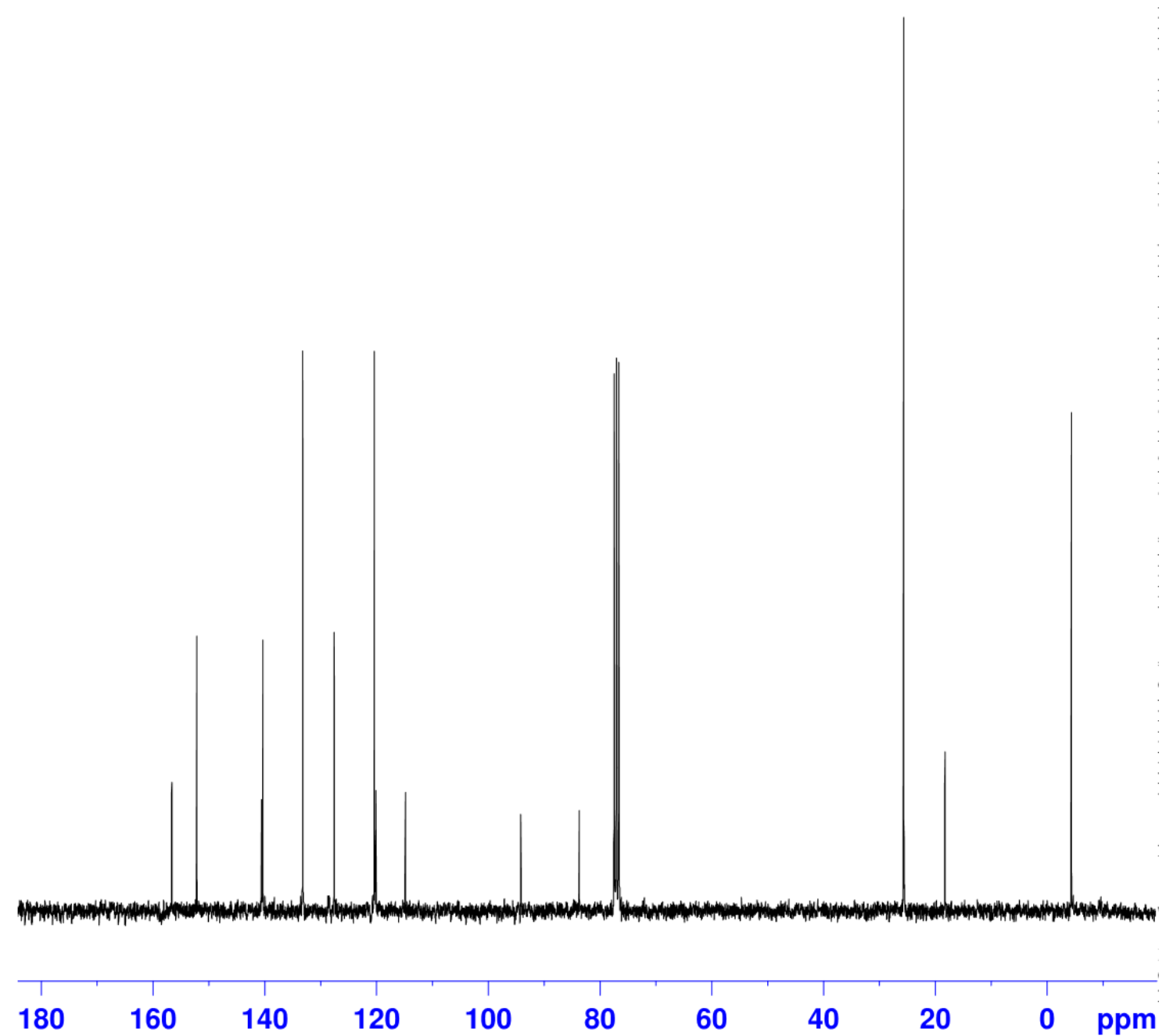


Figure S7. ${ }^{1} \mathrm{H}$ NMR Spectra of Compound 7

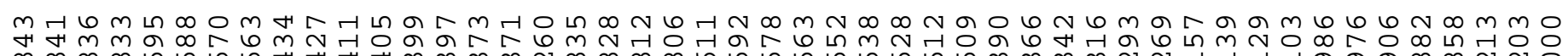

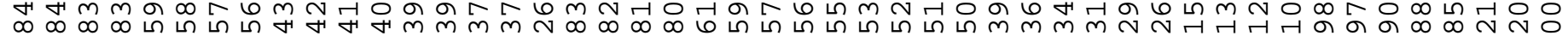

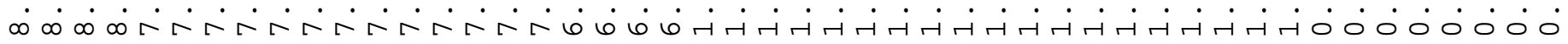
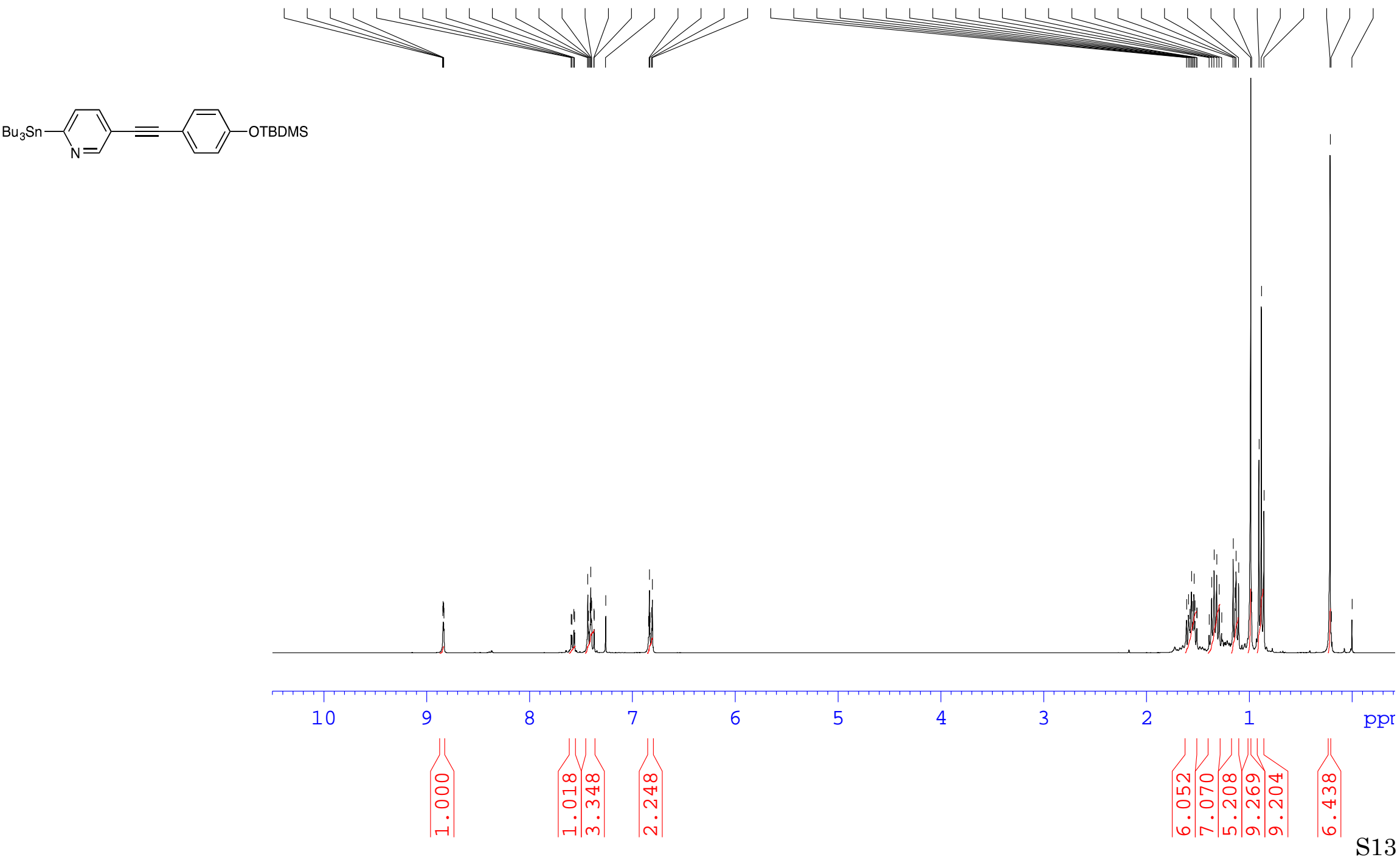
Figure S8. ${ }^{13} \mathrm{C}$ NMR Spectra of Compound 7

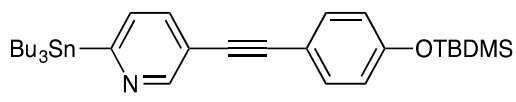

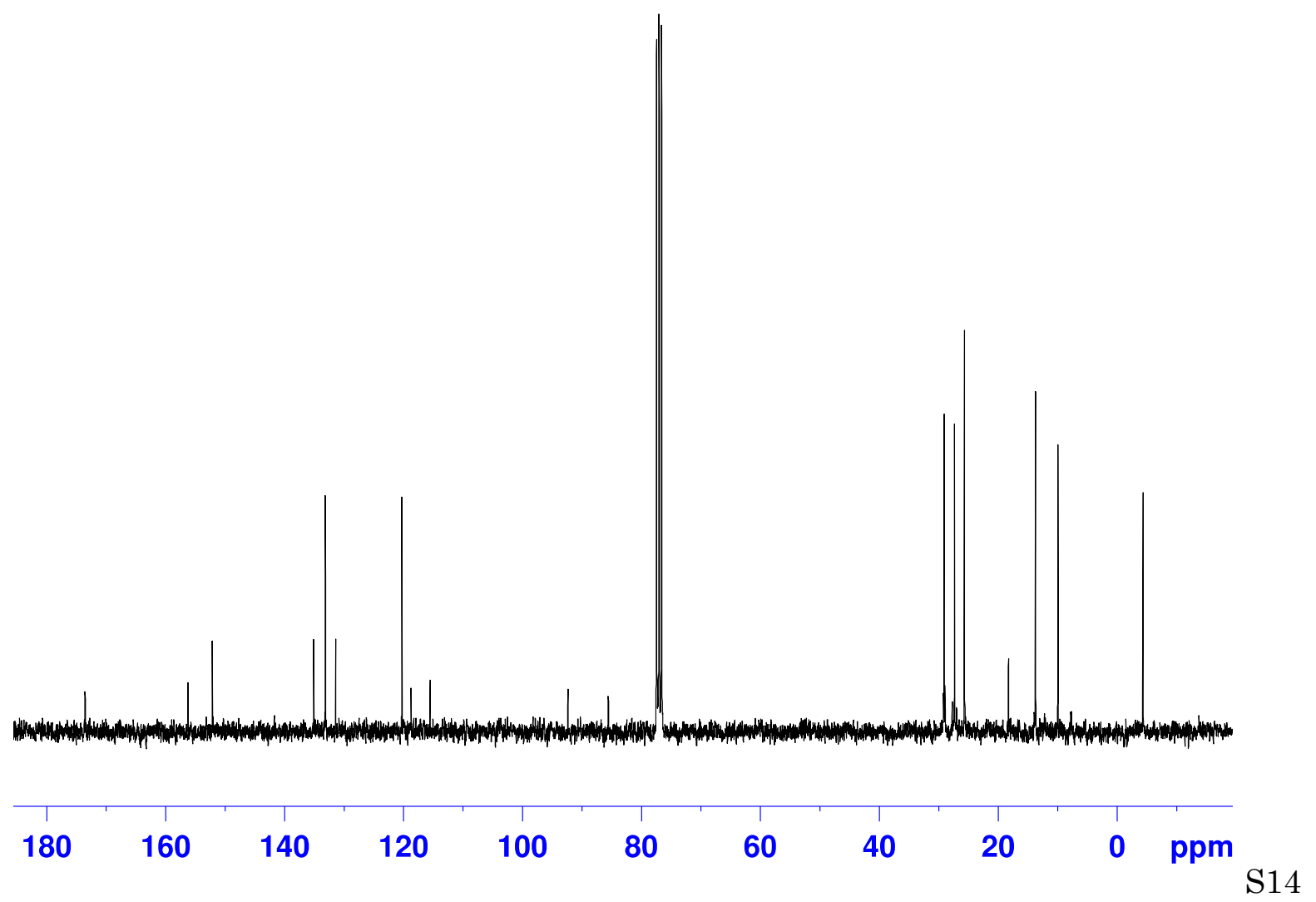


Figure S9. ${ }^{1} \mathrm{H}$ NMR Spectra of Compound 8

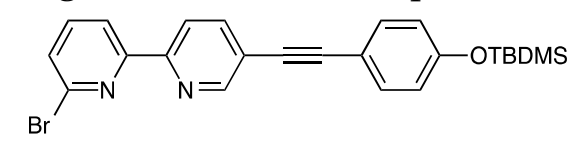

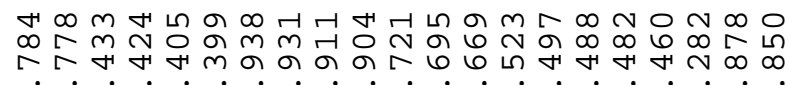

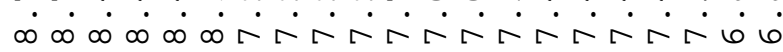

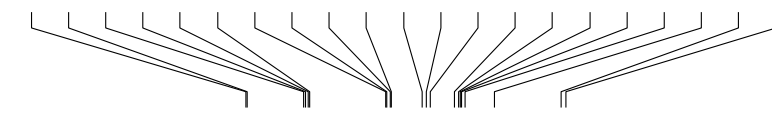

$\infty_{0}$

r.

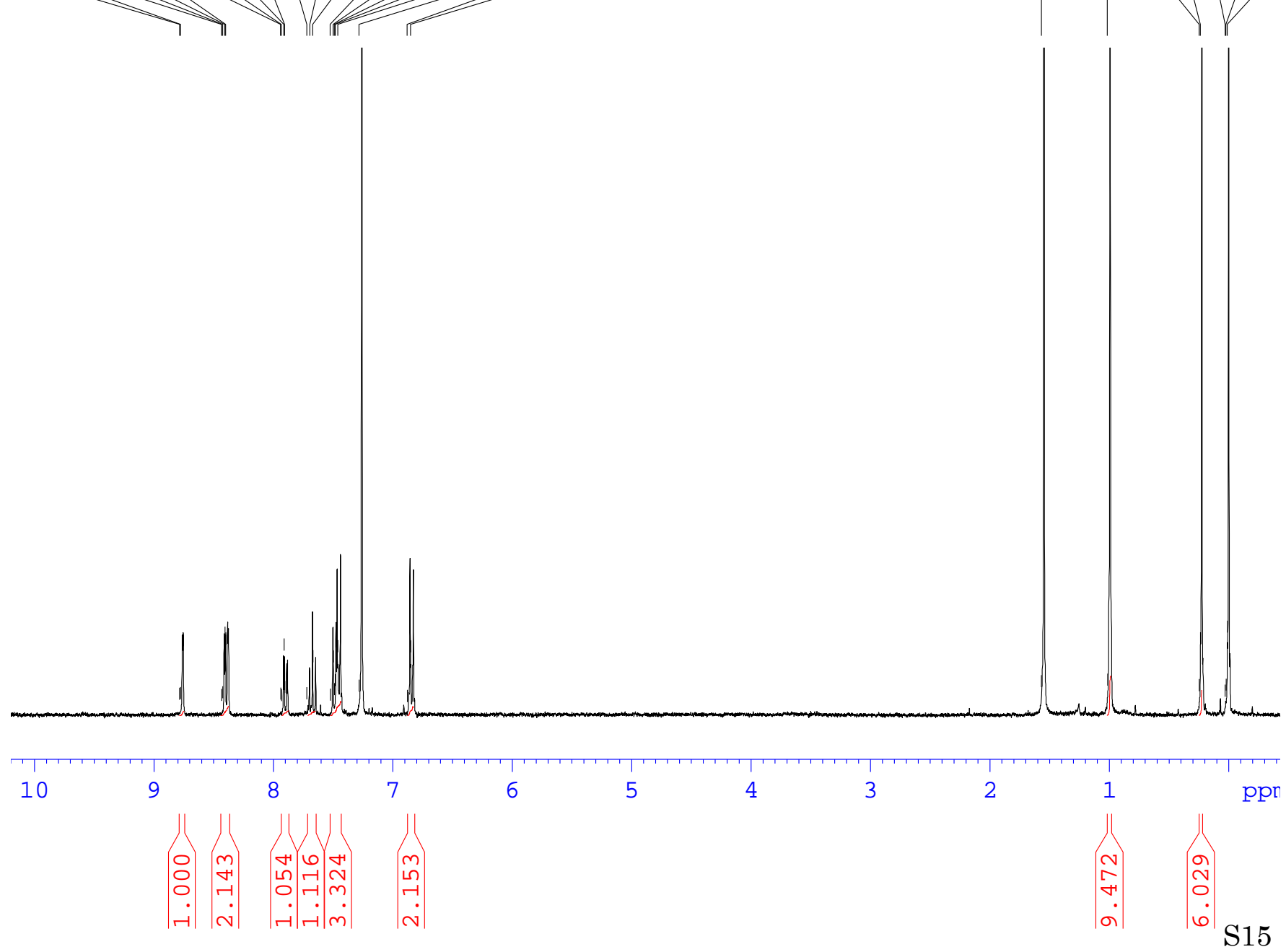


Figure S10. ${ }^{13} \mathrm{C}$ NMR Spectra of 8<smiles>CCCOc1ccc(C#Cc2ccc(-c3cccc(Br)n3)nc2)cc1</smiles>

\section{Compound}
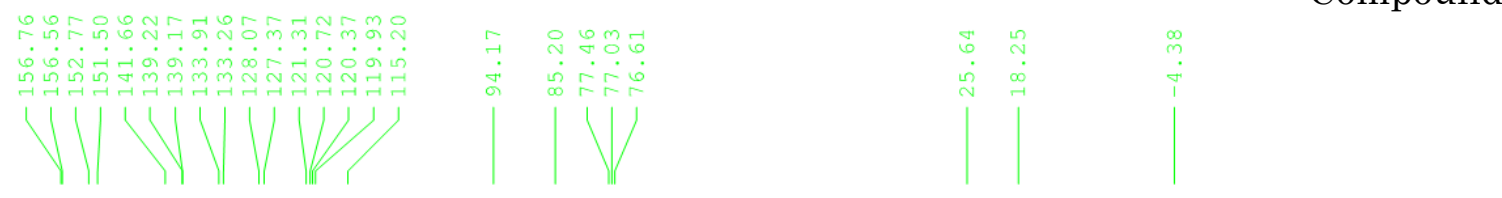

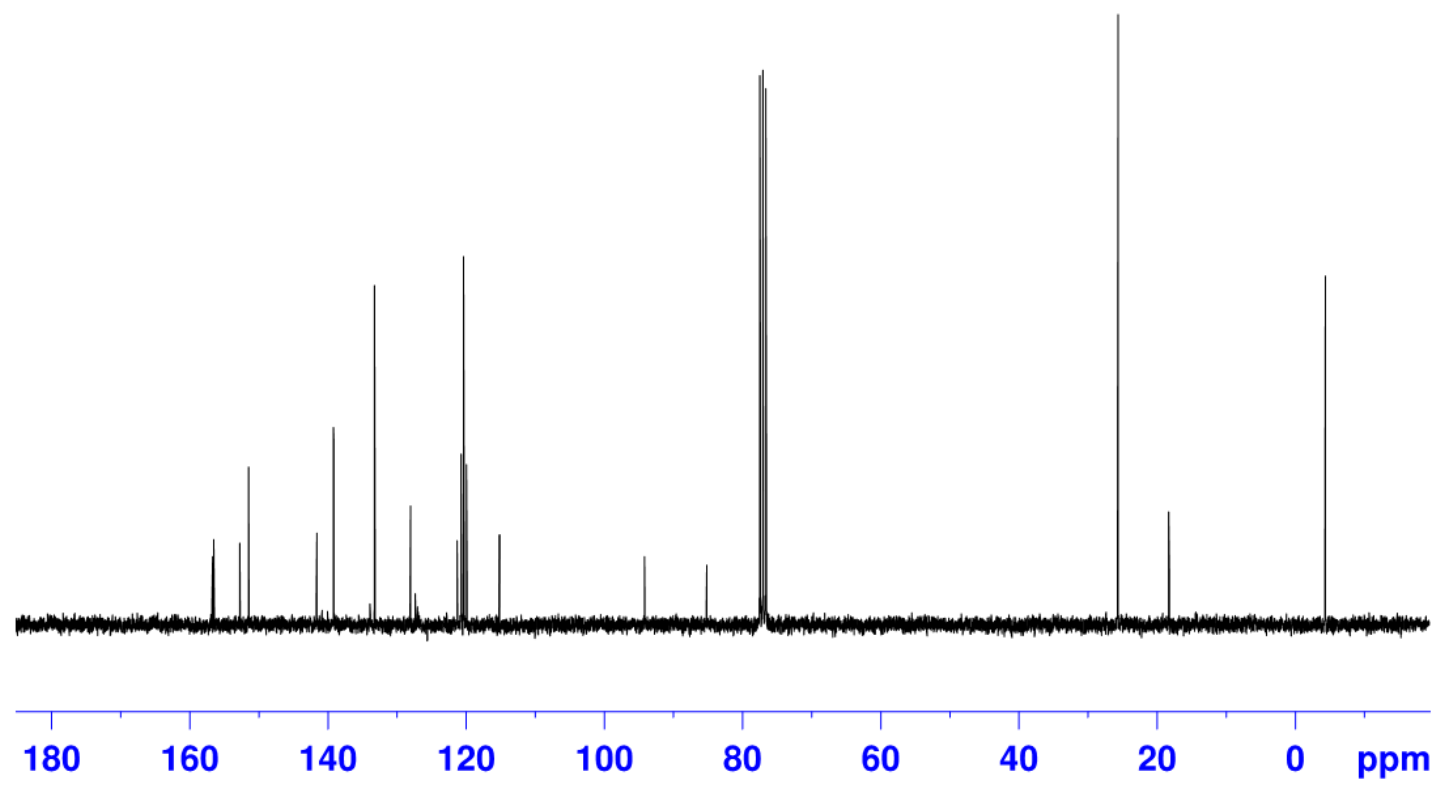


Figure S11. ${ }^{1} \mathrm{H}$ NMR Spectra of Compound 1

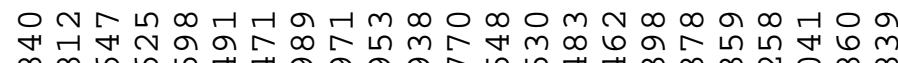

d
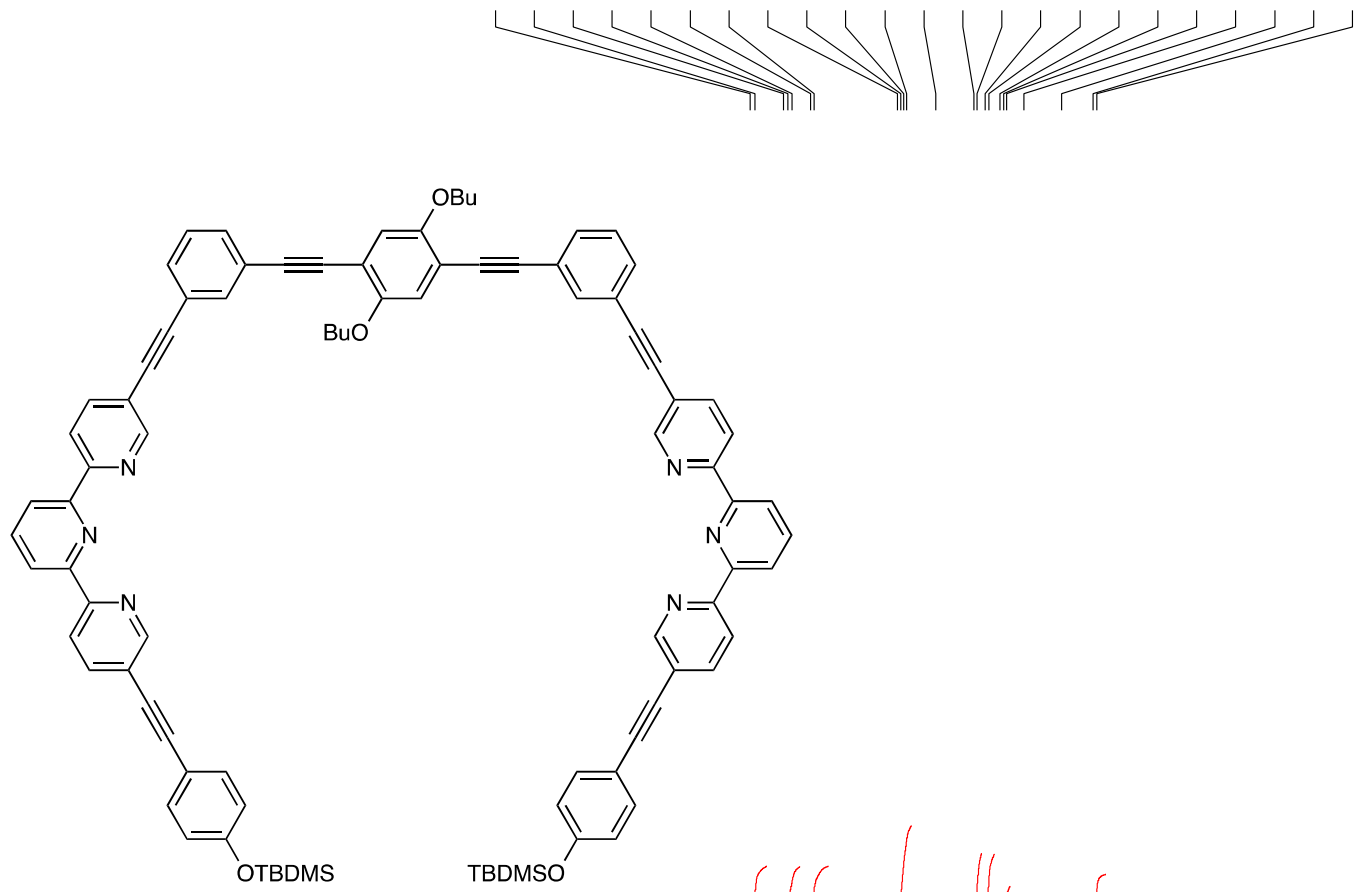

отвDMS

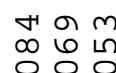

$\dot{\gamma} \dot{\gamma} \dot{\gamma}$

H ๑ ri ri

Lरो人l।

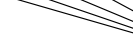

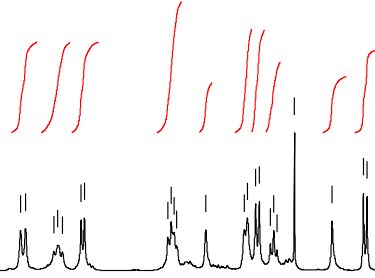
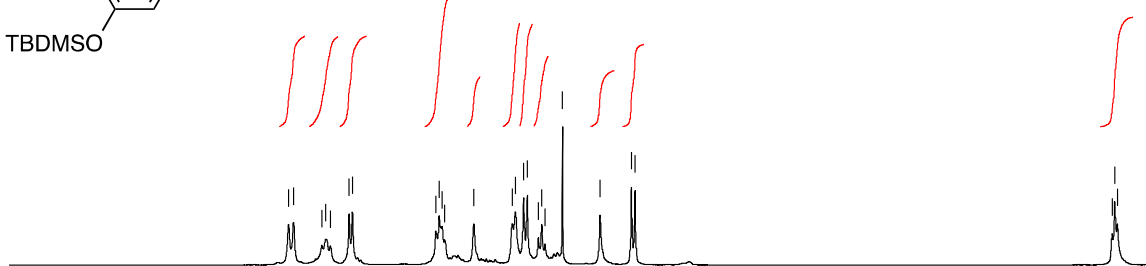

10

9

8

7

6

:8ñ 80 :

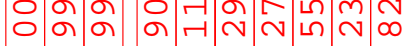

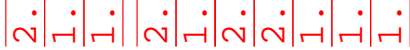

5

$\left|\begin{array}{c}4 \\ \\ \infty \\ 7 \\ y \\ \text { n. } \\ \mid\end{array}\right|$

3

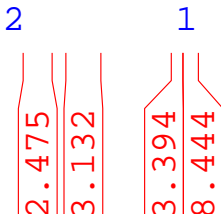

ppn

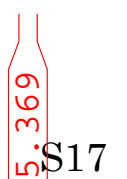


Figure S12. ${ }^{13} \mathrm{C}$ NMR Spectra of Compound 1

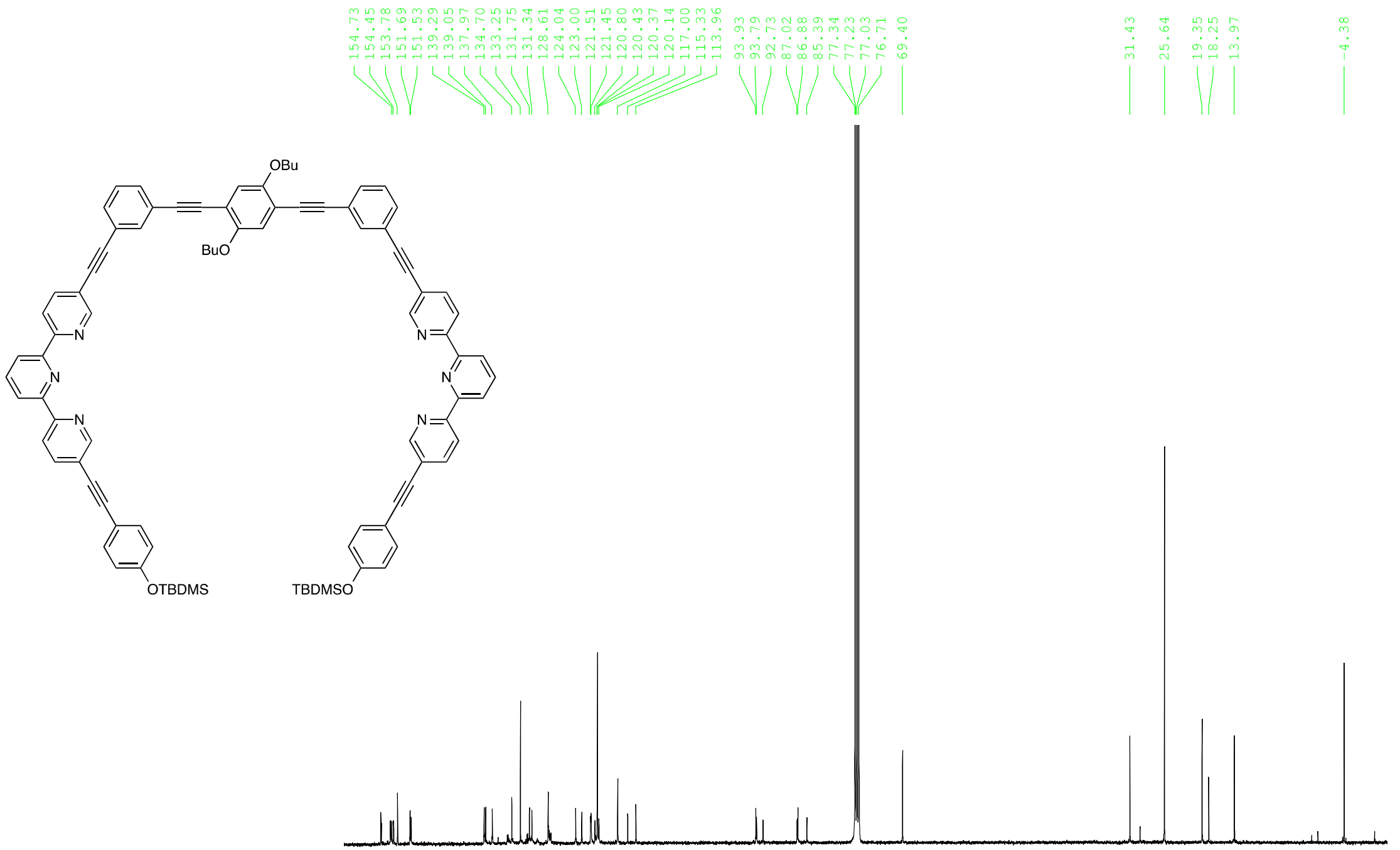

$\begin{array}{lllllllllllllllll}150 & 140 & 130 & 120 & 110 & 100 & 90 & 80 & 70 & 60 & 50 & 40 & 30 & 20 & 10 & 0 & \text { ppm }\end{array}$ 
Figure S13. ${ }^{13} \mathrm{C}$ NMR Spectra of Compound 1 (DEPT135)
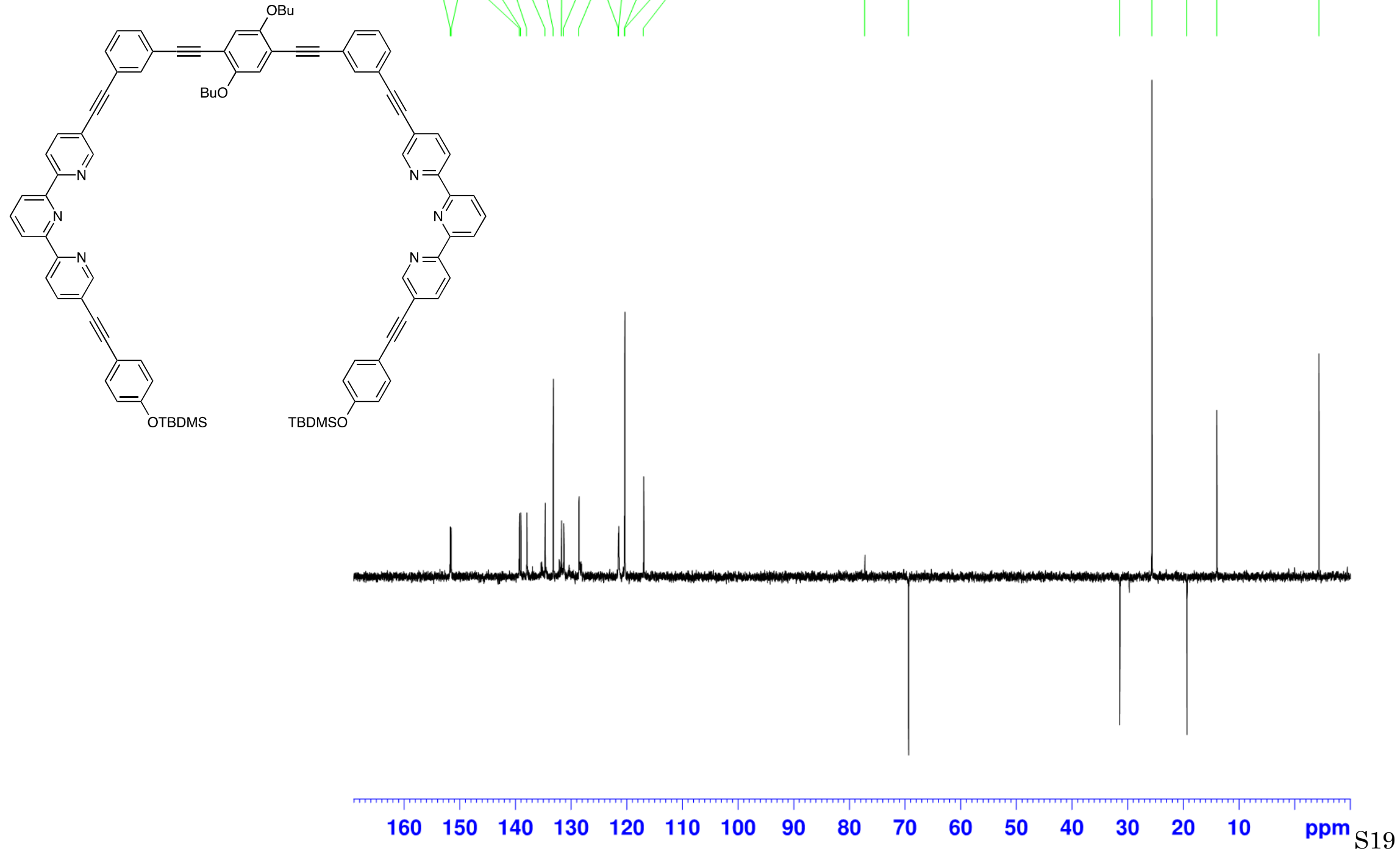
Figure S14. HH COSY Spectra of Compound 1
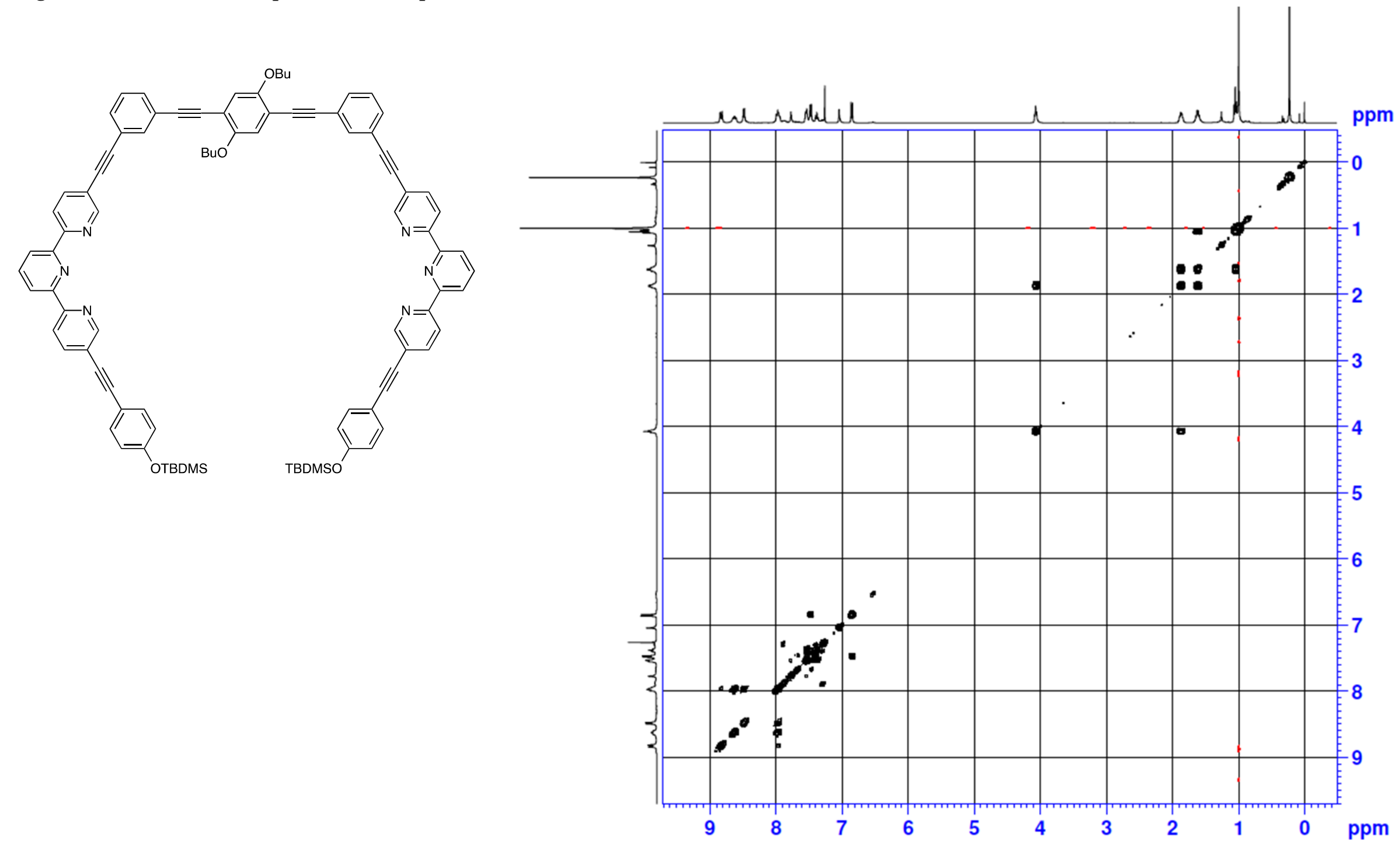
Figure S15. HSQC Spectra of Compound 1

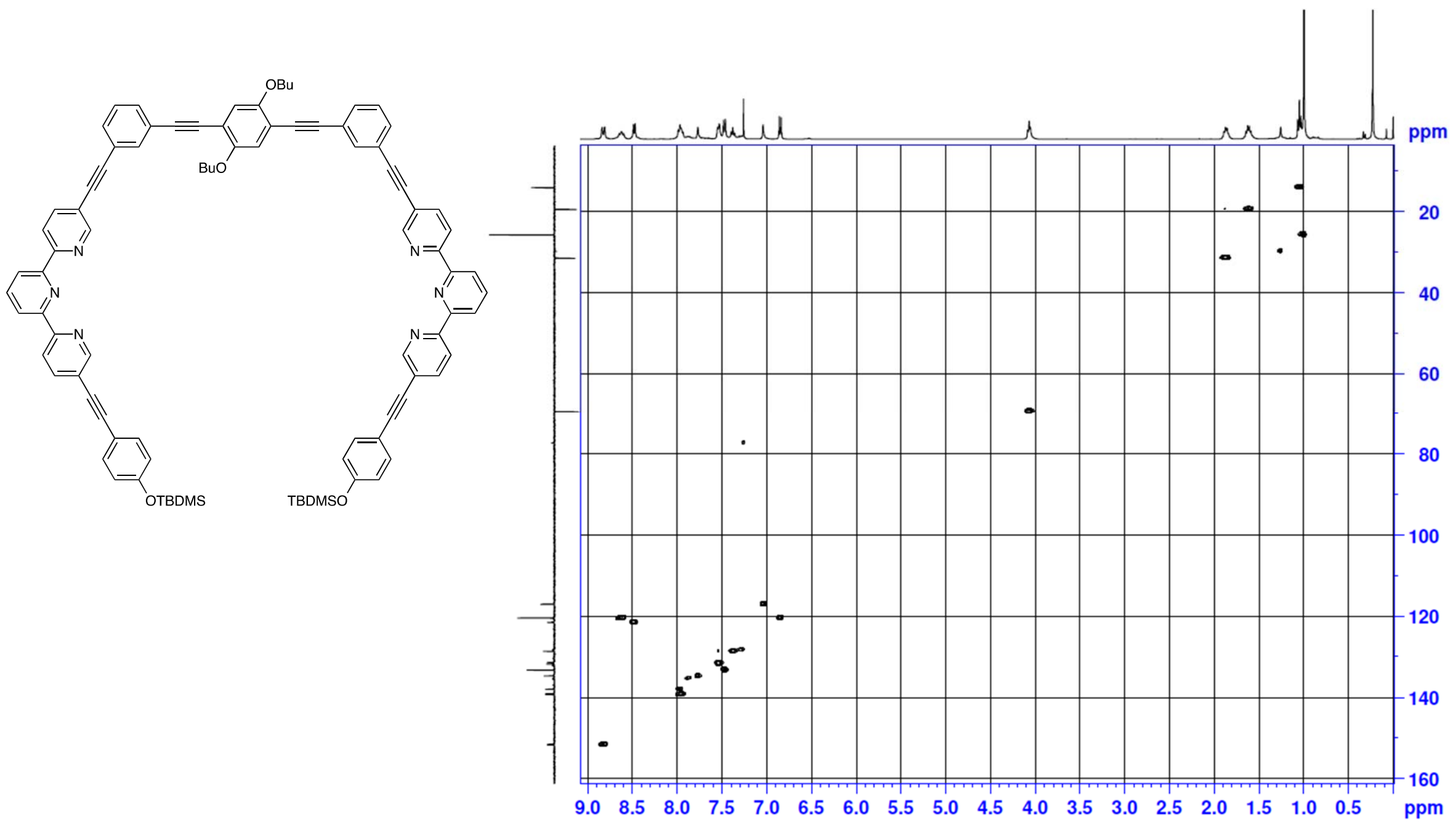


Figure S16. UV/Vis Titration of Compound 1 with $\mathrm{CoCl}_{2}$
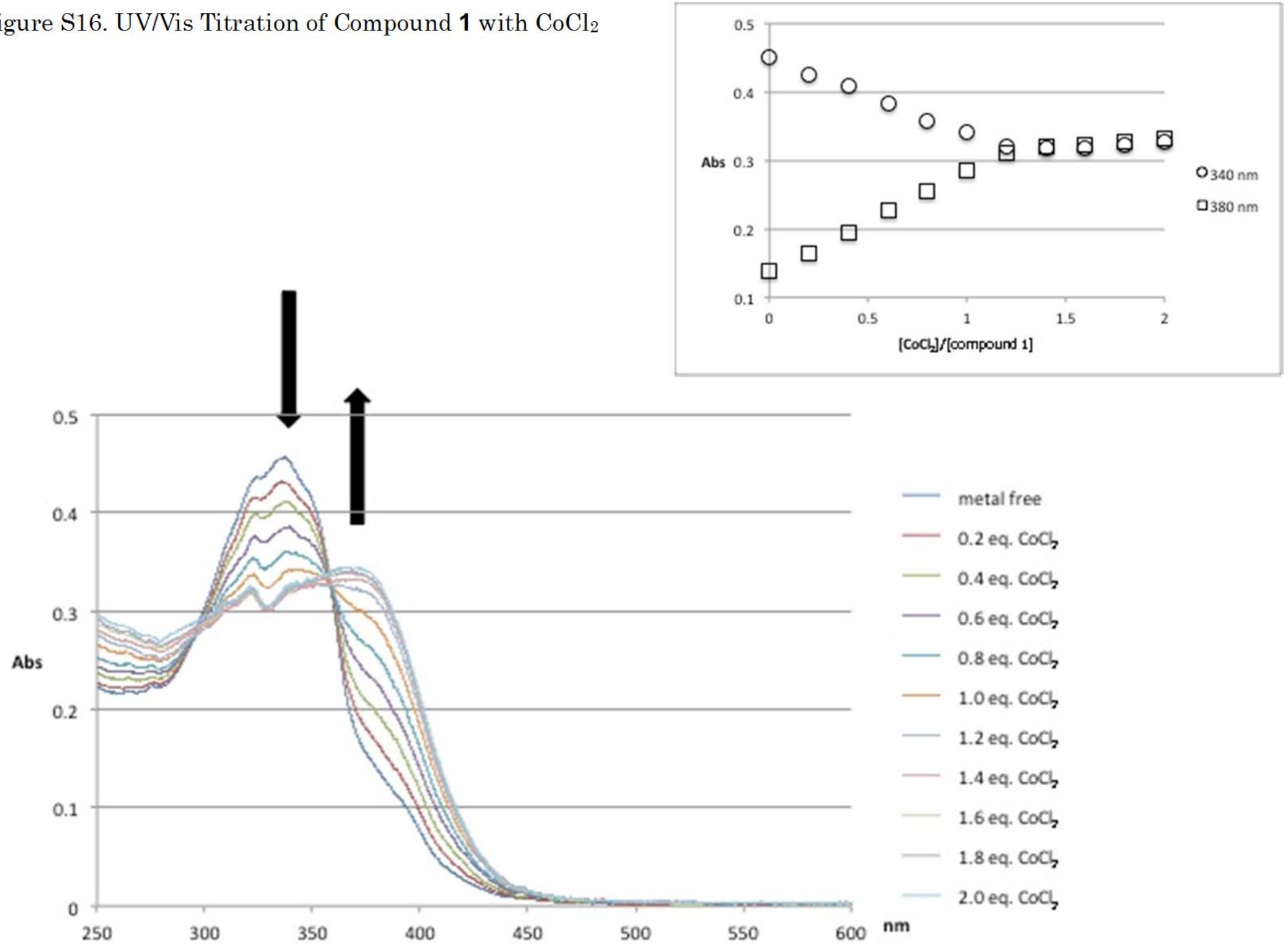
Figure S17. UV/Vis Titration of Compound 1 with $\mathrm{FeCl}_{2}$
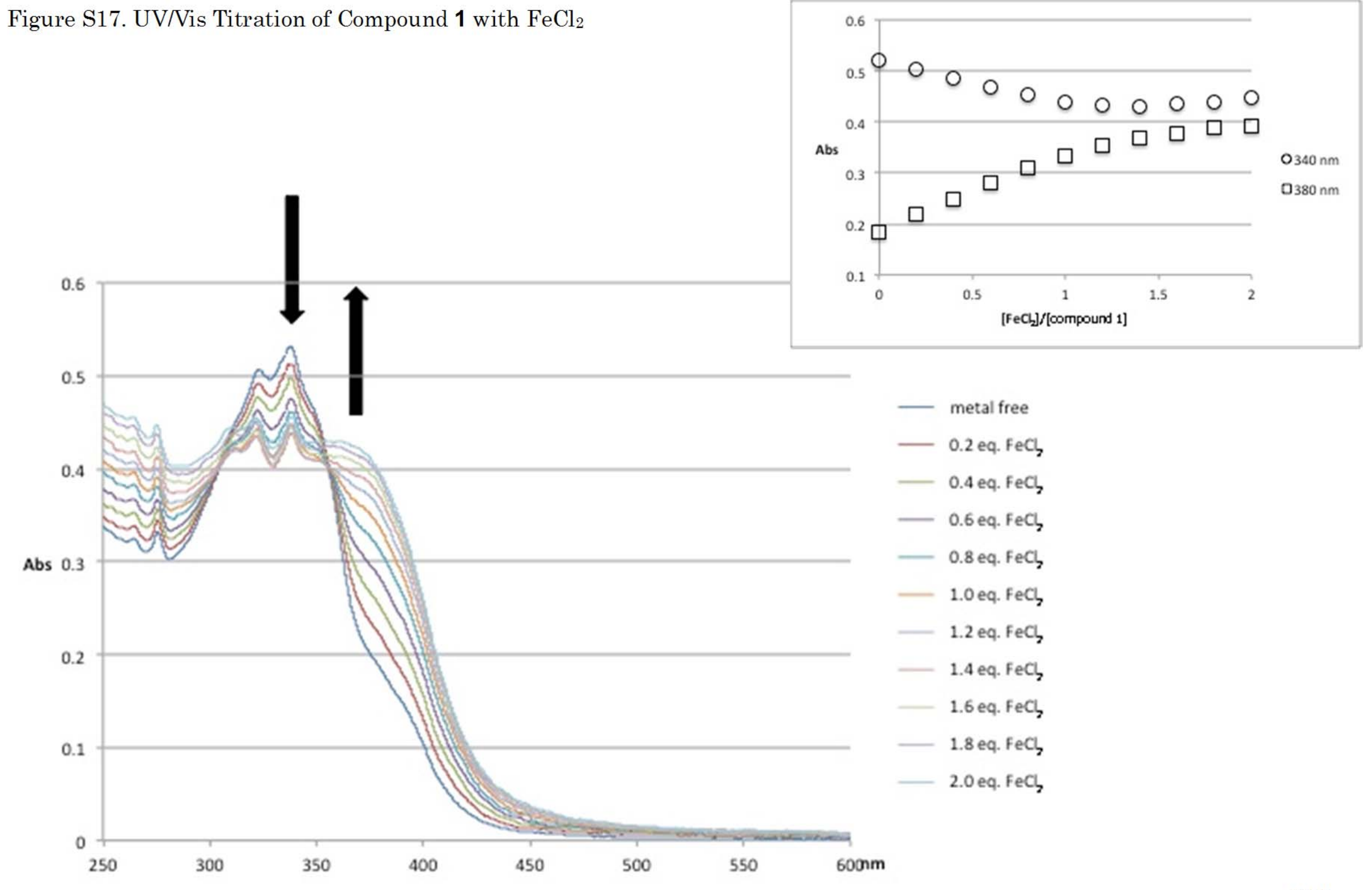
Figure S18. NMR Titration of Compound 1 with $\mathrm{ZnCl}_{2}$
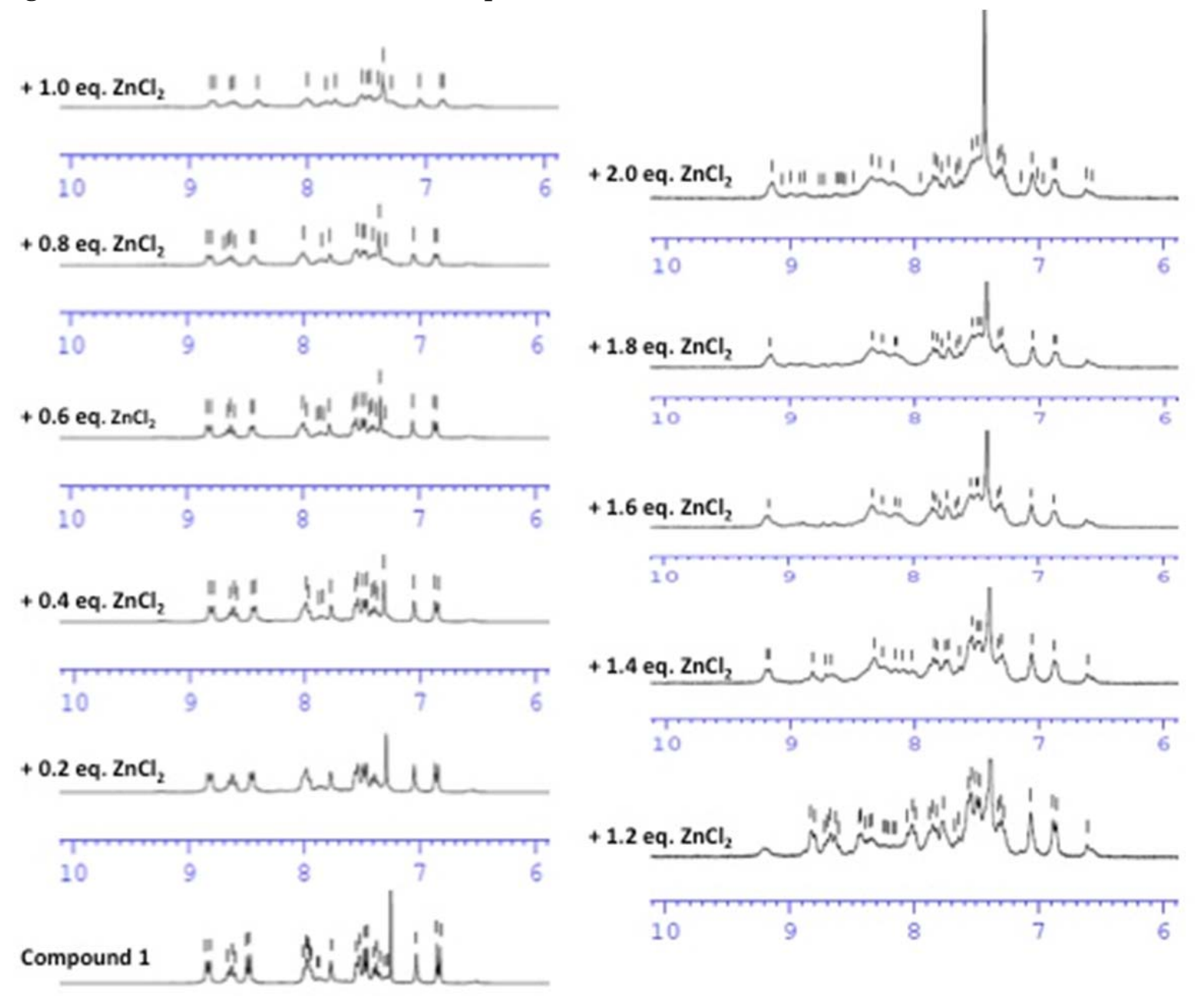

+1.4 eq. $\mathrm{ZnCl}_{2}$
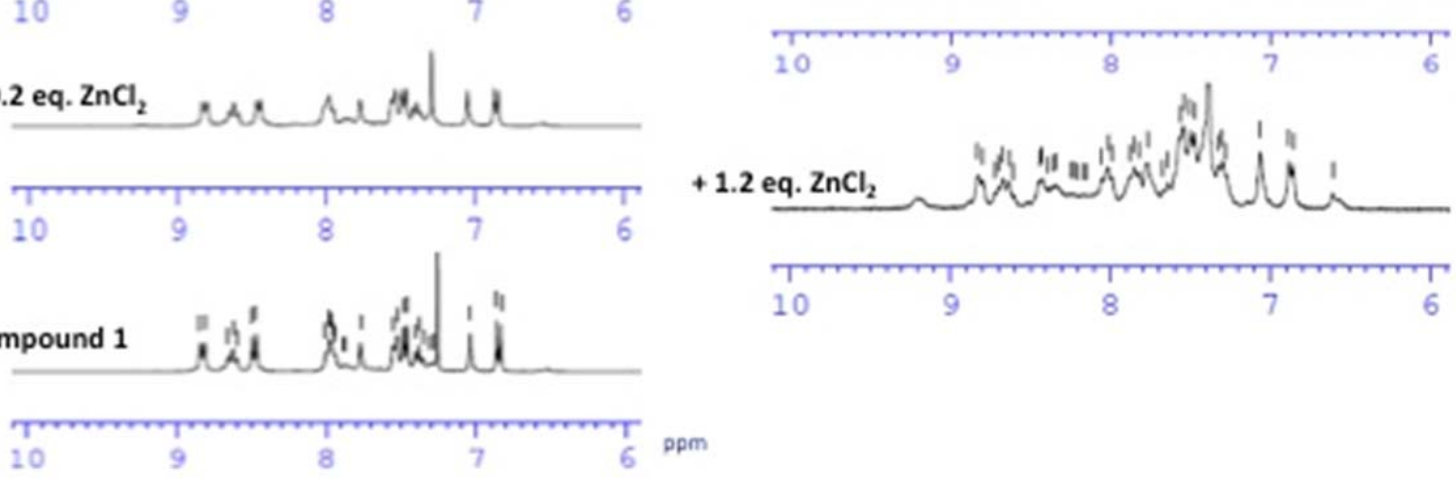
Figure S19. ESI-MS Spectra of Compound 1 with $\mathrm{ZnCl}_{2}$

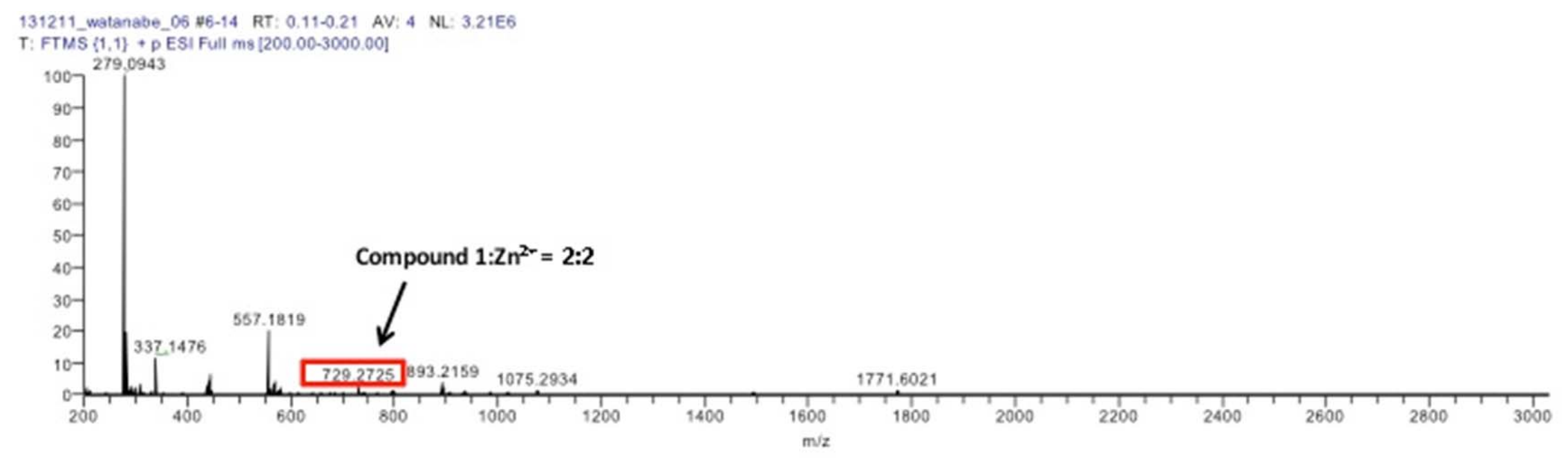

131211_watanabe_06 $\pm 6-14 \quad$ RT: $0.11-0.21$ AV: 4 NL: $4.73 E 4$

T: FTMS $\{1,1\}+$ D ESI Full $m 8$ [200.00-3000,00]

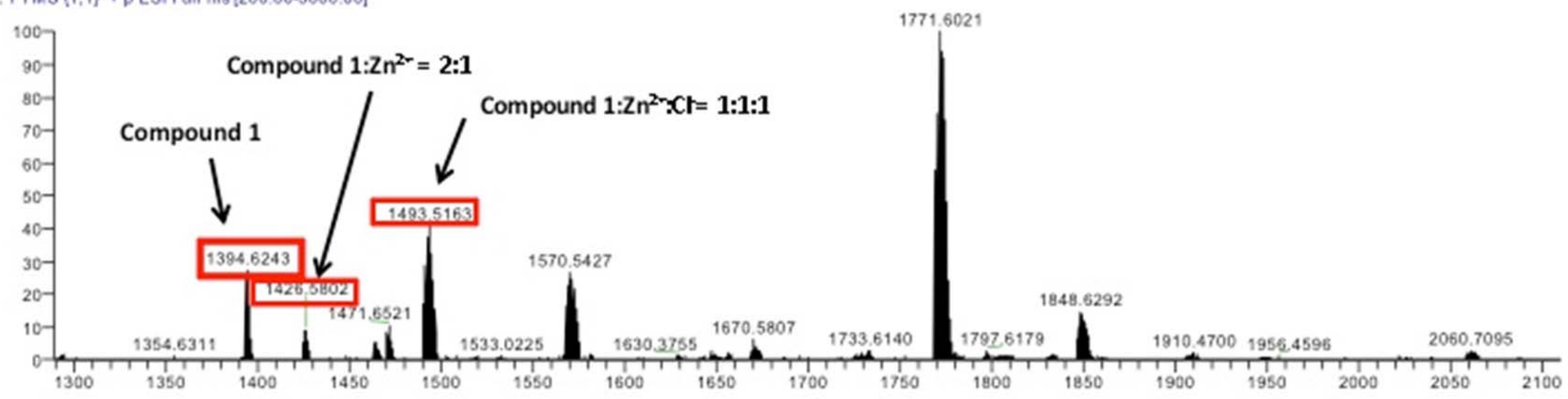

